\title{
Survival prediction in Amyotrophic lateral sclerosis based on MRI measures and clinical characteristics
}

\author{
Christina Schuster, Orla Hardiman and Peter Bede
}

\begin{abstract}
Background: Amyotrophic lateral sclerosis (ALS) a highly heterogeneous neurodegenerative condition. Accurate diagnostic, monitoring and prognostic biomarkers are urgently needed both for individualised patient care and clinical trials. A multimodal magnetic resonance imaging study is presented, where MRI measures of ALS-associated brain regions are utilised to predict 18-month survival.

Methods: A total of 60 ALS patients and 69 healthy controls were included in this study. $20 \%$ of the patient sample was utilised as an independent validation sample. Surface-based morphometry and diffusion tensor white matter parameters were used to identify anatomical patterns of neurodegeneration in $80 \%$ of the patient sample compared to healthy controls. Binary logistic ridge regressions were carried out to predict 18-month survival based on clinical measures alone, MRI features, and a combination of clinical and MRI data. Clinical indices included age at symptoms onset, site of disease onset, diagnostic delay from first symptom to diagnosis, and physical disability (ALSFRS-r). MRI features included the average cortical thickness of the precentral and paracentral gyri, the average fractional anisotropy, radial-, medial-, and axial diffusivity of the superior and inferior corona radiata, internal capsule, cerebral peduncles and the genu, body and splenium of the corpus callosum.
\end{abstract}

Results: Clinical data alone had a survival prediction accuracy of $66.67 \%$, with $62.50 \%$ sensitivity and $70.84 \%$ specificity. MRI data alone resulted in a prediction accuracy of $77.08 \%$, with $79.16 \%$ sensitivity and $75 \%$ specificity. The combination of clinical and MRI measures led to a survival prediction accuracy of $79.17 \%$, with $75 \%$ sensitivity and $83.34 \%$ specificity.

Conclusion: Quantitative MRI measures of ALS-specific brain regions enhance survival prediction in ALS and should be incorporated in future clinical trial designs.

Keywords: Amyotrophic lateral sclerosis, Magnetic resonance imaging, Biomarker, Diffusion tensor imaging, Cortical thickness, Binary logistic ridge regression, Cross-validation, Independent validation, Prognosis

\section{Background}

Amyotrophic lateral sclerosis is a relentlessly progressive neurodegenerative condition. While the clinical features of ALS are highly heterogeneous, the overall disease trajectory and life expectancy from diagnosis is relatively uniform, making it a template neurodegenerative condition for the development of diagnostic and prognostic biomarkers [1]. It is widely accepted that a

\footnotetext{
* Correspondence: bedep@tcd.ie

Quantitative Neuroimaging Group, Academic Unit of Neurology, Room 5.43, Biomedical Sciences Institute, Trinity College Dublin, Pearse Street, Dublin 2,
} Ireland

(c) The Author(s). 2017 Open Access This article is distributed under the terms of the Creative Commons Attribution 4.0 International License (http://creativecommons.org/licenses/by/4.0/), which permits unrestricted use, distribution, and reproduction in any medium, provided you give appropriate credit to the original author(s) and the source, provide a link to the Creative Commons license, and indicate if changes were made. The Creative Commons Public Domain Dedication waiver (http://creativecommons.org/publicdomain/zero/1.0/) applies to the data made available in this article, unless otherwise stated. long pre-symptomatic phase precedes the clinical bulbar or spinal symptoms at onset, but progresses to respiratory failure over time.

Clinical heterogeneity has multiple dimensions in ALS such as site of onset, coexisting cognitive and behavioural deficits, dominance of upper or lower motor neurodegeneration, variability in progression rates and the relatively distinct clinical profile of various ALS genotypes $[3,4]$. All of these variables make accurate individual prognostication particularly challenging. Clinical heterogeneity precludes smaller clinical trials [5] as a

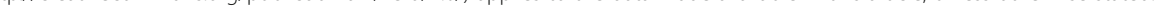


given drug may only be effective in certain ALS phenotypes. Robust and validated prognostic frameworks would enhance patient stratification into clinical trials, and enable the optimised management of individual patients. The planning and timing of supportive interventions such as feeding tube insertion, initiation of non-invasive ventilation, addressing end-of-life decisions and palliative measures could be guided by accurate prognostic markers.

Previous studies of ALS have successfully linked specific demographic and clinical variables to shorter survival; e.g. older age, bulbar or respiratory onset, recent symptom onset prior to diagnosis, significant motor impairment, coexisting executive dysfunction, rapid weight loss [6-9]. Attendance of a multidisciplinary ALS clinic has been linked to a better prognosis [10].

Magnetic resonance imaging (MRI) has been repeatedly proposed as a diagnostic or prognostic biomarker in ALS. [11, 12] The core imaging features of ALS related neurodegeneration are well described: degeneration of the precentral gyrus $[13,14]$, corpus callosum and corticospinal tract $[15,16]$. Despite numerous descriptive imaging studies in ALS, few studies have successfully translated group-level findings to aid the interpretation of individual data sets. While imaging measures were repeatedly proposed as potential diagnostic biomarkers in ALS, imaging parameters of single anatomical structures led to relatively poor diagnostic classification accuracy [17-20]. Imaging measures in ALS have also been explored as prognostic indicators. Neuronal integrity of the motor cortex has been directly linked to survival
[21] and corticospinal tract fractional anisotropy (FA) was used to predict 3-year survival [22].

The objective of this study is to develop and test an objective prognostic tool in ALS to predict 18-month survival based on quantitative MRI data. We hypothesised that structural MRI measures enhance prediction accuracy compared to clinical variables alone.

\section{Methods}

\section{Methods overview}

Patients were divided into a "training sample" and an "independent validation sample", to develop and test a binary logistic ridge regression model predicting 18month survival (Fig. 1). Core clinical parameters relating to survival were selected based on a comprehensive literature review. ALS-specific, MRI measures were selected based on group comparisons between the patients of the training cohort and healthy controls. Survival prediction accuracy was evaluated based on (a) clinical indices alone, (b) MRI measures alone and (c) based on both clinical and MRI measures.

\section{Participants}

Data were acquired prospectively as part of the biomarker initiative of the Academic Unit of Neurology in Trinity College. Written informed consent was provided by every participant in accordance to the Medical Ethics approval of the research project (Ethics (Medical Research) Committee - Beaumont Hospital, Dublin, Ireland). Inclusion criteria included a diagnosis of definite or probable ALS according to the revised El Escorial criteria [23] and the

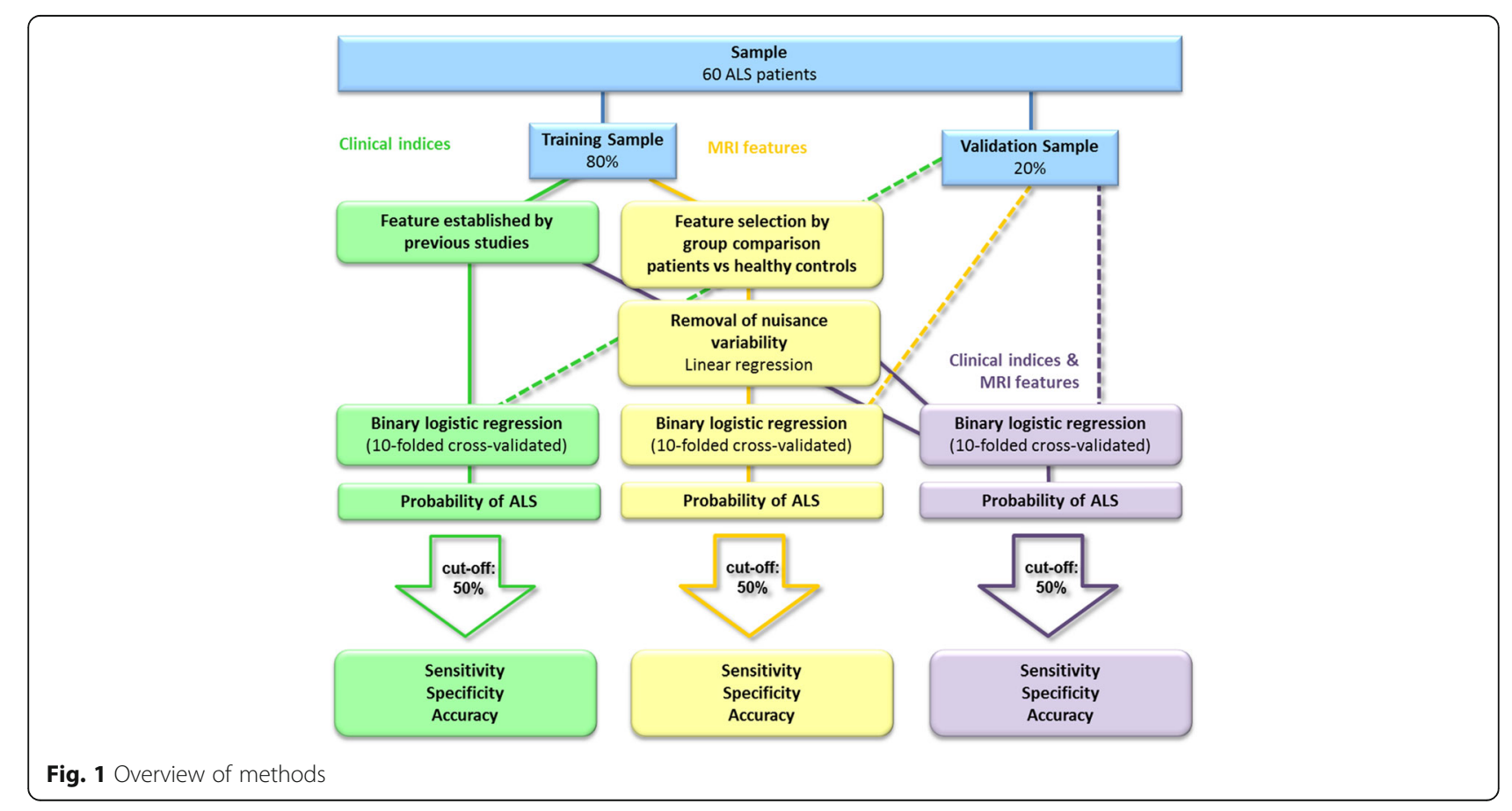


ability to lie supine in the MRI scanner for $45 \mathrm{~min}$. Exclusion criteria included unexpected radiological findings, coexisting psychiatric conditions, previous traumatic head injury, poorly controlled diabetes, hydrocephalus, prior haemorrhagic or ischaemic stroke. Patients with comorbid frontotemporal dementia according to the Rascovsky Criteria were also excluded because of the confounding imaging changes associated with this phenotype [24].

Based on the above criteria 69 consecutive ALS patients were prospectively enrolled and scanned between January 2011 and January 2015. Out of the 69 patients 3 patients could not tolerate the duration of the MRI protocol, and unexpected imaging findings were identified in 3 patients; falcine meningioma, gliotic cortical changes secondary to an old head injury, and a small old parietal stroke. Survival data was reviewed based on the national ALS-register in June 2016. Out of the 63 patients included, 33 were alive more than 18 months after their scan and 30 patients passed away within 18 months. To provide equally sized study groups of short and long survivors, 30 patients were randomly selected out of the 33 patients who were alive 18 months after their scans. $80 \%$ of the patients were randomly allocated to the training sample and $20 \%$ to the validation sample. In order to highlight the ALS specific pathology, the training sample was compared to an age- and gender matched group of 69 healthy controls. [25] The demographic and clinical profile of the participants in presented in Table 1.

\section{Imaging data acquisition}

MR data were acquired on a 3 Tesla Philips Achieva MRI platform with a maximum gradient strength of $80 \mathrm{mT} / \mathrm{m}$ using an 8 -channel receive-only head coil. T1-weighted images were obtained using a three- dimensional inversion recovery prepared spoiled gradient recalled echo (IR-SPGR) sequence with a field of new (FOV) of $256 \times 256 \times 160 \mathrm{~mm}$, spatial resolution $=1 \mathrm{~mm} 3$, TR $/ \mathrm{TE}=8.5 / 3.9 \mathrm{~ms}$,TI $=1060 \mathrm{~ms}$, flip angle $=8^{\circ}$, SENSE factor $=1.5$. Diffusion Tensor Images (DTI) were acquired using a spin-echo planar imaging (SEEPI) sequence with a 32-direction Stejskal-Tanner diffusion encoding scheme: FOV $=245 \times 245 \times 150 \mathrm{~mm}$, spatial resolution $=.5 \mathrm{~mm} 3,60$ slices with no interslice gap, TR/ $\mathrm{TE}=7639 / 59 \mathrm{~ms}$, SENSE factor $=2.5$, b-values $=0$, $1100 \mathrm{~s} / \mathrm{mm} 2$, with SPIR fat suppression and dynamic stabilisation in an acquisition time of $5 \min 41 \mathrm{~s}$.

\section{Imaging data analyses \\ MRI pre-processing}

White matter (WM) analysis The pre-processing of the diffusion weighted images included eddy current corrections, motion corrections, and brain-tissue extraction in FSL [26]. A diffusion tensor model was fitted at each voxel, generating maps of fractional anisotropy (FA), mean diffusivity (MD), axial diffusivity (AD), and radial diffusivity (RD). Each dataset was aligned to the FMRIB58a_FA standard-space images. Next, a mean FA image was created. Each subject's aligned FA data was then projected onto the FMRIB58a_FA standard-space skeleton and the resulting data fed into voxel-wise cross-subject statistics.

Cortical thickness (CT) analysis Cortical thickness was evaluated using the FreeSurfer imaging analysis suite (http://surfer.nmr.mgh.harvard.edu/; version 5.3.0), which has been both validated using histological [27] and manual measurements [28]. The automated processing pipeline consists of skull-stripping, registration, intensity

Table 1 Clinical and demographic data of study participants

\begin{tabular}{|c|c|c|c|c|c|c|c|c|}
\hline & & & Training Samp & & & Validation Sam & iple & \\
\hline & Healthy co & trols & ALS patients $\mathrm{S}$ & urviving & & ALS patients $s$ & urviving & \\
\hline & & $p$-value* & $<18$ months & $>18$ months & $p$-value & $<18$ months & $>18$ months & $p$-value \\
\hline $\bar{n}$ & 69 & & 24 & 24 & & 6 & 6 & \\
\hline Gender (male/ female) & $34 / 35$ & $P=0.22$ & $17 / 7$ & $13 / 11$ & $P=.37$ & $3 / 3$ & $2 / 4$ & $P=1$ \\
\hline Handedness (right/left) & $64 / 5$ & $P=.55$ & $23 / 1$ & $20 / 4$ & $P=.35$ & $5 / 1$ & $5 / 1$ & $P=1$ \\
\hline Age, years (means, SD**) & $59.97(9.9)$ & $P=.17$ & $63.18(8)$ & $61.76(10.73)$ & $P=.60$ & $63.96(8.03)$ & $55.09(8.82)$ & $P=.09$ \\
\hline Site of onset (bulbar/spinal) & & & $8 / 16$ & $10 / 14$ & $P=.76$ & $3 / 3$ & $2 / 4$ & $P=1$ \\
\hline Diagnostic delay, years (mean, SD) & & & $1.2(0.81)$ & $1.05(0.75)$ & $P=.51$ & $1.28(1.1)$ & $0.88(0.3)$ & $P=.43$ \\
\hline $\begin{array}{l}\text { Disease duration from symptom onset until } \\
\text { scan, years (mean, SD) }\end{array}$ & & & $2.17(1.01)$ & $2.32(1.34)$ & $P=.67$ & $1.94(1.44)$ & $1.85(0.54)$ & $P=.89$ \\
\hline ALSFRS-r (mean, SD) & & & $34.48(6.84)$ & $37.38(6.21)$ & $P=.12$ & $34.5(8.34)$ & $39.17(4.17)$ & $P=.25$ \\
\hline King's College Staging (1/2/3/4/ unavailable) & & & $4 / 5 / 3 / 7 / 5$ & $4 / 9 / 5 / 4 / 2$ & & $0 / 1 / 2 / 2 / 1$ & $2 / 2 / 1 / 1 / 0$ & \\
\hline MITOS Staging (0 /1/ 2/ 3/ unavailable) & & & 10/8/1/0/5 & $19 / 2 / 0 / 1 / 2$ & & $3 / 1 / 1 / 0 / 1$ & $6 / 0 / 0 / 0 / 0$ & \\
\hline Survival from scan, years (mean, SD) & & & $0.94(0.32)$ & $2.26(1.11)$ & $P<.01$ & $0.92(0.26)$ & $2.62(1.32)^{* *}$ & $P<.05$ \\
\hline
\end{tabular}

*healthy controls were compared to the training sample of 48 patients. ${ }^{*} S D$ standard deviation 


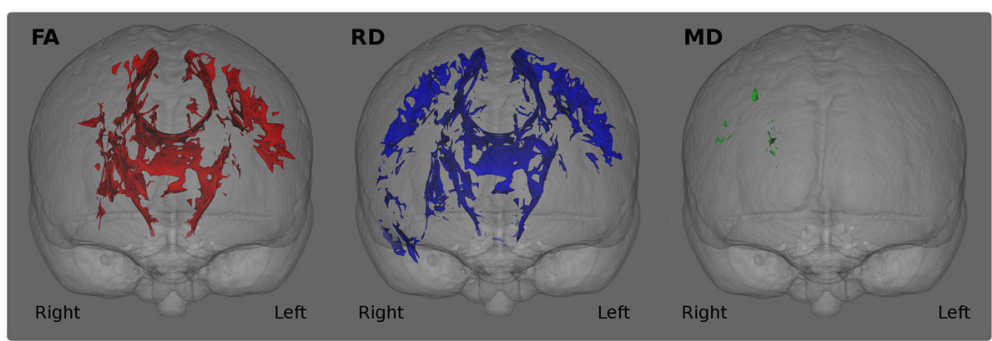

Fig. 2 White matter analyses. Group comparisons between ALS patients and controls. The significance level is set to $p<.01$ FWE

normalization, Talairach transformation, tissue segmentation, and surface parcellation. Tissue segmentation determines the white/grey matter interface (white matter surface) and grey matter/cerebrospinal boundary (pial surface). The result of this process was individually reviewed, and if errors were detected the segmentation step was repeated. Cortical thickness has been defined as the distance (vertex) from the white matter surface to the nearest point on the pial surface.

\section{Feature selection}

In order to identify ALS-specific pathological brain regions, patients of the training sample were compared to healthy controls using age as a nuisance variable. The significance level for the group comparisons were set to $p<0.01$ corrected using family-wise error (FWE). Figure 2. Based on these analyses, the following core white matter regions were selected as discriminatory features for the binary ridge regression: the superior corona radiata, inferior corona radiata, anterior and posterior limbs of the internal capsule, cerebral peduncles (mesencephalic crus) and the genu, body and splenium of the corpus callosum. Figure 3. These regions were defined based on the above patientsversus-controls contrast using the JHU DTI-based white-matter atlas labels. The average value of each DTI index (i.e. FA, RD, MD, AD) was extracted from each white matter region.

The comparative cortical thickness analyses were significant at $p<.05$ corrected for multiple comparisons using false discovery rate (FDR). Figure 4. Cortical features were selected based on these comparisons and expanded to include the entire precentral gyrus and the paracentral gyrus based on available literature [29, 30]. The Desikan-Killiany atlas [31] was utilised to define the cortical regions and the average cortical thickness was extracted from both regions.

For all variables, values in left and right hemisphere were averaged. The pre-processing steps of the independent validation sample were analogous to the pre-processing pipeline of the training sample.

\section{Reducing age-related variability}

The effect of aging on MRI measures is well established and it has been shown that classification accuracy may be improved by removing this nuisance variable. The confounding effect of age is particularly important in ALS which affects a fairly a wide age range. From an imaging perspective, a young patient with severe physical disability may exhibition similar brain changes to older patients with less advanced disease. Moreover, age in ALS is considered a prognostic factor. [8, 32] To account for age-related variability the method of Koikkalainen et al. was implemented [33]. A linear regression model was fitted to the distribution of the values of each feature of the control group using age as independent variable. Based on this equation, we estimated the predicted value for each feature for each individual subject. These values were then subtracted from the measured values resulting in age-corrected measurements for each feature.

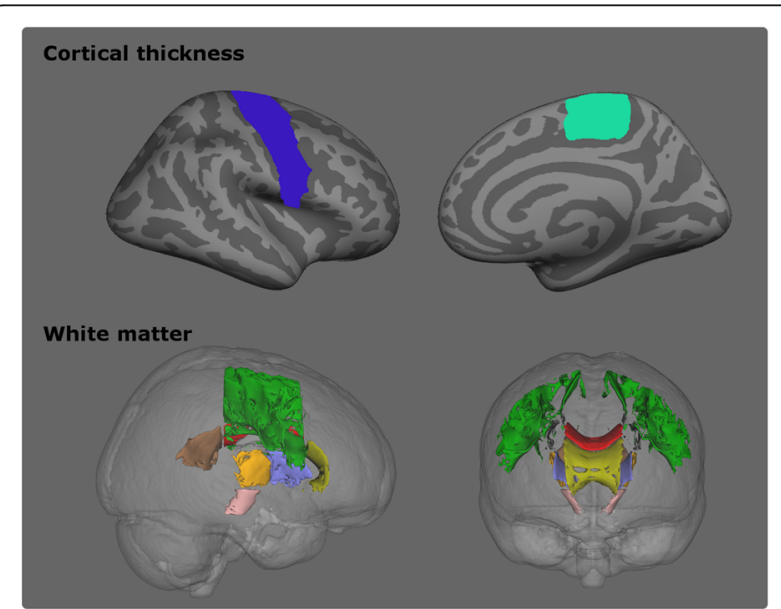

Fig. $3 \mathrm{MRl}$ features included in the regression analyses. Top: Cortical thickness. Blue: precentral gyrus, Light green: paracentral gyrus. Only the right hemisphere is displayed. Bottom: White matter. Green: superior corona radiata, grey: corona radiata, orange: posterior limb of the internal capsule, lilac: anterior limb of the internal capsule, rose: cerebral peduncle, yellow: genu of corpus callosum, red: body of the corpus callosum, brown: splenium of the corpus callosum 


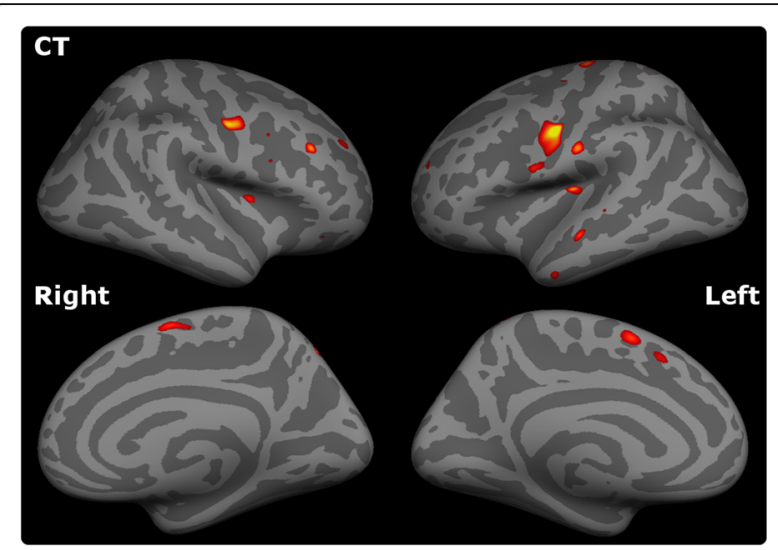

Fig. 4 Cortical thickness analyses. Group comparison between ALS patients and controls. The significance level is set to $p<.05$ FDR

\section{Statistical analyses}

Three binary logistic ridge regressions were fitted using (a) clinical indices alone (b) MRI features alone (c) and both clinical and MRI parameters. Clinical features included age at disease onset, site of disease onset (bulbar/ spinal), diagnostic delay (time interval from symptom onset to diagnosis), and ALSFRS-r at the time of the scan. Table 2. MRI features corrected for age-related variability consisted of the average cortical thickness of the precentral gyrus, the average FA, RD, MD and $\mathrm{AD}$ of the superior corona radiata, inferior corona radiata, anterior and posterior limbs of the internal capsule, cerebral peduncles and the genu, body and splenium of the corpus callosum. Figure 3. The outcome variable was the probability of surviving less than 18 months. The statistical software $R$ [34] and the package 'glmnet' $(\alpha=0)$ [35] was utilised to carry out the logistic ridge regression. The tuning parameter $\lambda$ was selected based on ten-folded cross-validation which was repeated 100 times. The model with the smallest misclassification

Table 2 List of clinical and MRI features

\begin{tabular}{|c|c|}
\hline Clinical indices & MRI features \\
\hline - Age at disease onset & Cortical thickness \\
\hline - Site of disease onset & - Precentral gyri \\
\hline - Diagnostic delay & - Paracentral gyri \\
\hline \multirow[t]{8}{*}{ - Disease severity ALSFRS-r } & $\begin{array}{l}\text { White matter } \\
\text { - Superior corona radiata }\end{array}$ \\
\hline & - Inferior corona radiata \\
\hline & - Anterior limbs of the internal capsule \\
\hline & - Posterior limbs of the internal capsule \\
\hline & - Cerebral peduncles \\
\hline & - Genu of the corpus callosum \\
\hline & - Body of the corpus callosum \\
\hline & - Splenium of the corpus callosum \\
\hline
\end{tabular}

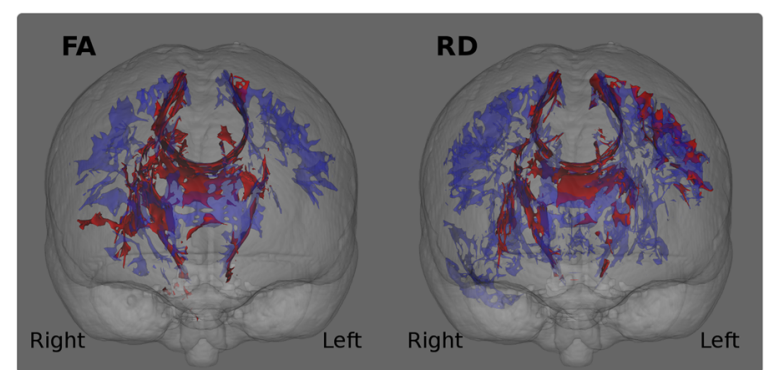

Fig. 5 Patterns of white matter pathology in short and long survivors. Blue colour indicates white matter degeneration in 'short survivals' (<18 months) in comparison to controls, and red colour indicates white matter degeneration in 'long survivors' (>18 months) in comparison to controls at $p<0.05$ (FWE) correcting for age

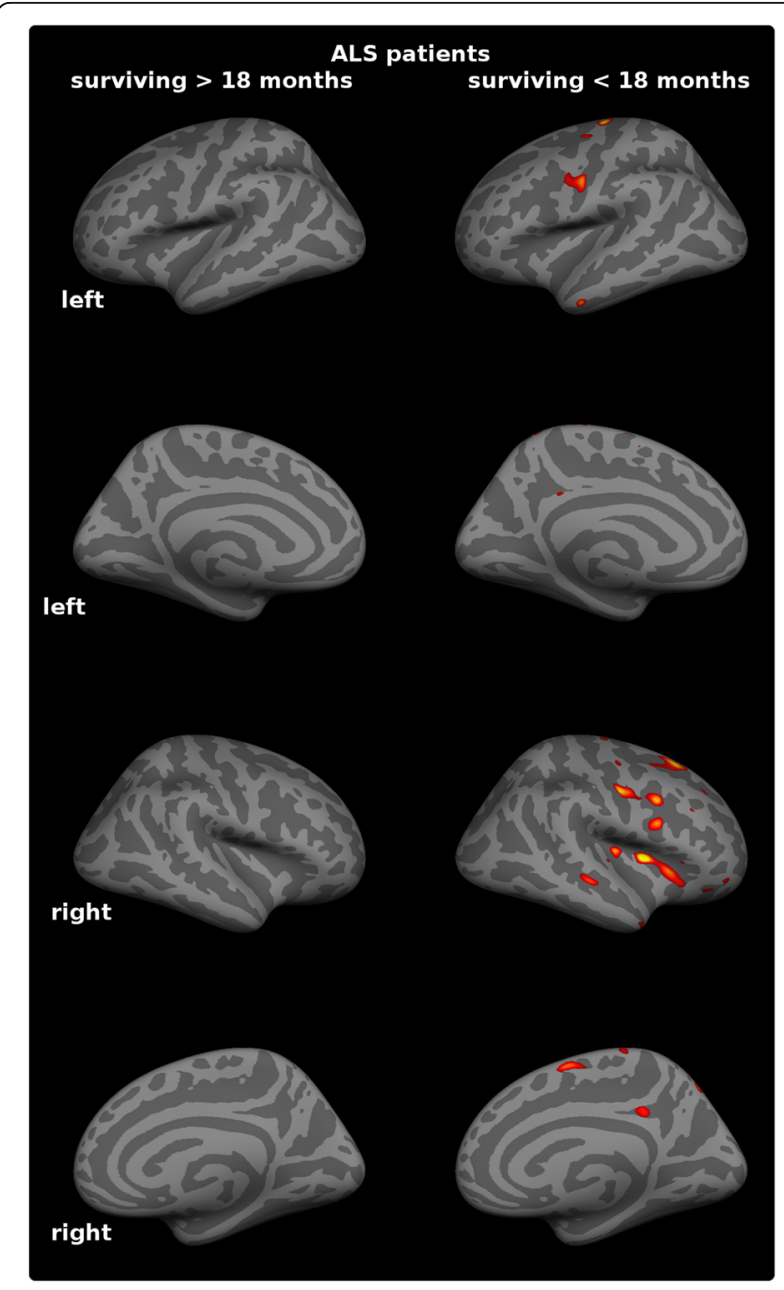

Fig. 6 Patterns of cortical pathology in short and long survivors. Extensive, multifocal grey matter pathology is identified in 'short survivals' ( $<18$ months) compared to controls at $p<0.05$ (FDR) which is not captured at this statistical threshold in long-survivors. (>18 months) 
error averaged over the 100 estimations was selected. Subsequently, the ridge regression algorithm was used to estimate the probability of each participant in the validation sample to belong to the group surviving less than 18 months after the brain scan.

\section{Results}

\section{Group comparisons}

The comparison of ALS patients in the training sample and controls highlighted ALS-specific patterns of neurodegeneration, i.e. cortical thinning of the precentral and paracentral gyri (Fig. 4) and white matter degeneration of all segments of the corticospinal tract and corpus callosum. (Figure 2). The direct comparison of patients surviving more than 18 months and those surviving less than 18 months, did not reach statistical significance with family-wise error corrections. However, patients with shorter survival have demonstrated significantly more widespread cortical and white matter changes in comparison to controls than those surviving longer Figs. 5 and 6.

Figure 7 shows the probability of each patient to survive less than 18 months based on (a) clinical parameters, (b) MRI measures, and (c) both clinical and MRI

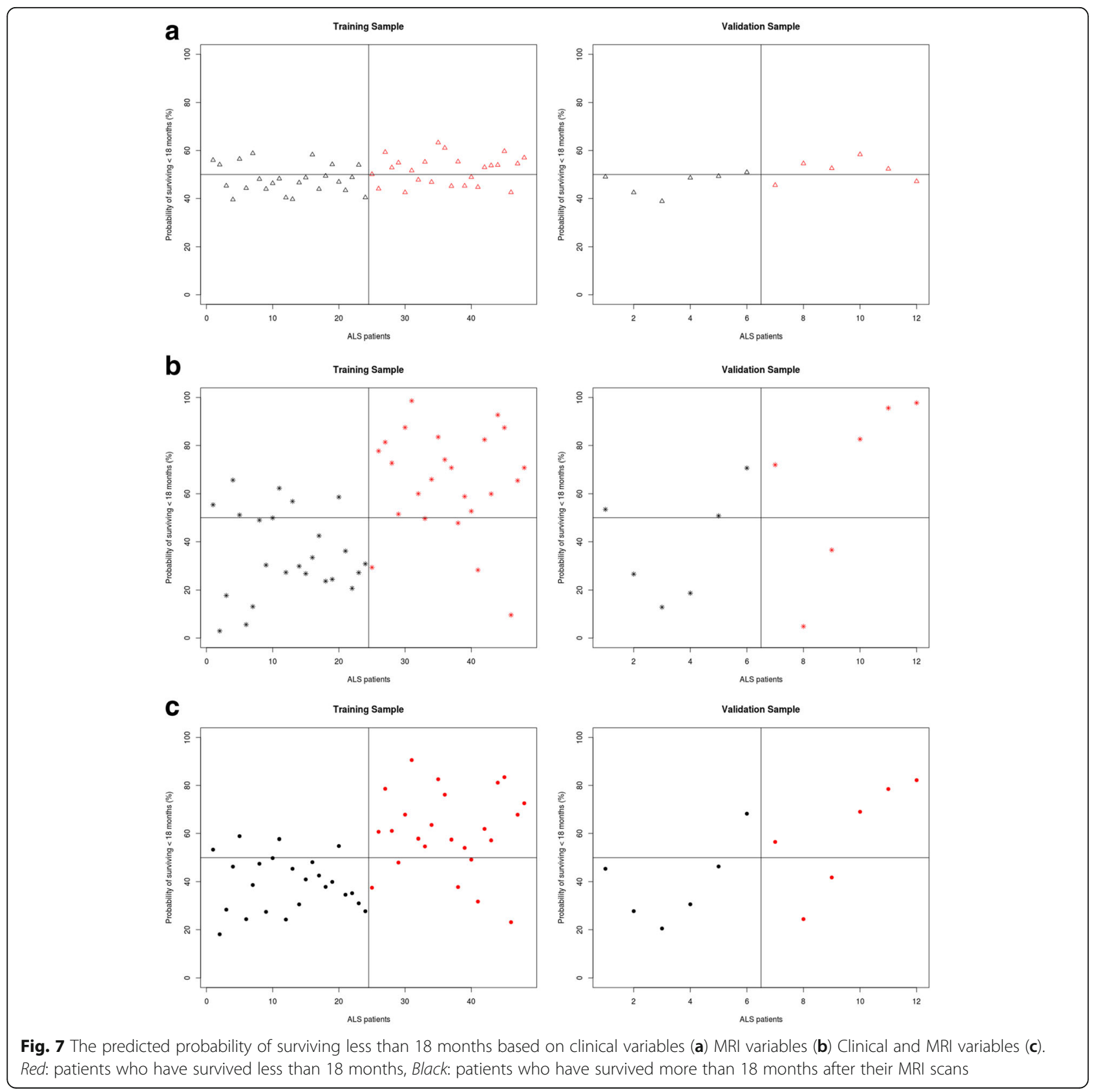


indices. Using a cut-off score of $50 \%$, the accuracy, sensitivity and specificity of prognostic classification in presented in Table 3. The coefficient estimates for each regression are presented in Additional file 1: Table S1.

Analysis of misclassification revealed that among the clinical variables the total ALSFRS-R showed a significant difference between correctly classified and

Table 3 Classification outcomes using a 50\% cut-off probability

A, Classification based on clinical characteristics alone

Training sample

$\begin{array}{lllll}\text { Predicted class } & \begin{array}{l}\text { Survival } \\ <18 \text { months }\end{array} & \begin{array}{l}\text { Survival } \\ >18 \text { months }\end{array} & \text { Sensitivity } & 62.50 \% \\ \begin{array}{l}\text { True Class Survival } \\ <18 \text { months }\end{array} & 15 & 7 & \text { Specificity } & 70.84 \% \\ \begin{array}{l}\text { Survival } \\ >18 \text { months }\end{array} & 9 & 17 & \text { Accuracy } & 66.67 \% \\ \begin{array}{l}\text { Validation sample } \\ \text { Predicted class }\end{array} & \text { Survival } & \text { Survival } & \text { Sensitivity } & 66.67 \% \\ \begin{array}{l}\text { True Class Survival } \\ <18 \text { months }\end{array} & 4 & >18 \text { months } & & \\ \begin{array}{l}\text { Survival } \\ >18 \text { months }\end{array} & 2 & 1 & \text { Specificity } & 83.34 \% \\ & & 5 & \text { Accuracy } & 75.00 \%\end{array}$

B, Classification based on MRI measures alone

Training sample

Predicted class Survival Survival Sensitivity $79.16 \%$

$<18$ months $>18$ months

True Class Survival $19 \quad 6$

$<18$ months

Survival

$5 \quad 18$

$>18$ months

Validation sample

Predicted class Survival Survival Sensitivity $66.70 \%$ $<18$ months $>18$ months

True Class Survival 4

$<18$ months

Survival

$>18$ months

2

C, Classification based on clinical and MRI measures

Training sample

\begin{tabular}{lllll} 
Predicted class & $\begin{array}{l}\text { Survival } \\
<18 \text { months }\end{array}$ & $\begin{array}{l}\text { Survival } \\
>18 \text { months }\end{array}$ & Sensitivity & $75.00 \%$ \\
$\begin{array}{l}\text { True Class Survival } \\
<18 \text { months }\end{array}$ & 18 & 4 & Specificity & $83.34 \%$ \\
$\begin{array}{l}\text { Survival } \\
>18 \text { months }\end{array}$ & 6 & 20 & Accuracy & $79.17 \%$ \\
$\begin{array}{l}\text { Validation sample } \\
\text { Predicted class }\end{array}$ & $\begin{array}{l}\text { Survival } \\
<18 \text { months }\end{array}$ & $\begin{array}{l}\text { Survival } \\
\text { (18 months }\end{array}$ & Sensitivity & $66.67 \%$ \\
True ClassSurvival & 4 & 1 & Specificity & $83.34 \%$ \\
$<18$ months & & 5 & Accuracy & $75.00 \%$ \\
\hline Survival $>18$ months & 2 & 5 & & \\
\hline
\end{tabular}

misclassified patients. When predicting a survival of less than 18 months, false negative patients had a higher ALSFRS-R in comparison to false positives who had lower ALSFRS-R scores (average ALSFRS-R for True Positive $=30.93(\mathrm{SD}=6.39)$, False Negative $=40.11$ $(\mathrm{SD}=2.03), p<.01)$. Additional file 1: Table S2-13.

\section{Discussion}

The presented study explores the role of MRI measures as prognostic biomarkers in ALS. While diagnostic and monitoring biomarkers have been extensively investigated in ALS, there is a scarcity of prognostic studies. One of the key finding of the study is the more widespread white and grey matter degeneration in 'short-survivors' compared to 'long-survivors'. Figures 5 and 6 . Based on these results, we aimed to systematically evaluate the value of structural MRI measures of diseasedefining brain regions and clinical indices in predicting the probability of 18-month survival.

Based on the combination of structural brain measures and clinical characteristics, mortality within 18-months was predicted with relatively high accuracy; $79.17 \%$. Moreover, $83.3 \%$ of patients were correctly identified as surviving for longer than 18 months following their brain scan, and $75 \%$ of the sample was correctly identified as surviving less than 18-months. Applying the regression algorithm to an independent validation sample further supports the validity of these findings. Despite the relatively small sample size of the validation cohort, the algorithm reached $75 \%$ accuracy. $83.34 \%$ of patients were correctly identified as surviving more than 18months and $66.67 \%$ of patients were correctly identified as surviving less than 18 months. Based on MRI measures alone, the accuracy and sensitivity of the classification was similar, but the patients surviving more than 18 months were less likely to be identified correctly.

Using clinical and demographic measures alone, without MRI indices, prediction accuracy was considerably lower $(66.67 \%)$. Similarly, the sensitivity and specificity profile of these predictions were inferior to the ones also incorporating MRI measures. These findings underscore the benefit of MRI measures of ALS-associated brain regions in predicting 18-months survival. (Table 3.)

Evaluating misclassified patients based on clinical features alone, the group incorrectly classified surviving less than 18 months was significantly less physically impaired. They had a higher score of the ALSFRS-R. In contrast, patients misclassified as surviving longer than 18 months, had significantly longer disease duration. Adding MRI measure, there was no difference found between misclassified patients, again emphasizing the benefit of this additional information.

Previous studies have linked MRI measures to survival. Two-year survival was predicted using motor cortex 
spectroscopy with a sensitivity of $67 \%$ and a specificity of $83 \%$ [21] and corticospinal tract diffusivity changes were utilised to predict three-year survival with a specificity of $61.5 \%$ and accuracy of $71.0 \%$. [22] In contrast to previous studies, we present a multi-modal approach, assessing cortical thickness alterations in addition to the four most commonly used indices of white matter degeneration. Additionally, we test the generalisability of our classification method in an independent validation sample.

Accurate prognostic markers have a role in clinical management as well as in clinical trials. In the absence of effective disease-modifying therapies, the optimal timing of supportive measures [36], end-of-life decisions [37], palliative interventions [38] is particularly important in ALS. While ALS patients are eager to participate in clinical trials in all stages of the disease, it may be desirable to enrol relatively homogenous patient cohorts soon after their diagnosis, when limited neurodegenerative change has taken place. $[2,5]$ It is frequently the case however that heterogeneous patient cohorts are enrolled in clinical trials, encompassing diverse phenotypes in order to rapidly meet the targeted sample size [39]. In the era of precision medicine, therapeutic strategies and clinical trial designs should be tailored to specific phenotypes [40, 41] and disease stages [39]. For example, stem cells therapy is regarded to be less successful in bulbar onset ALS patients, and patients with advanced disease [42]. Clinical trials of specific phenotypes or homogenous cohorts may have other advantages, such as the inclusion of patients who are likely to progress at a relatively uniform rate. It has been proposed that the inclusion of patients with rapid progression rates may shorten clinical trials [43].

Using objective, validated and observer-independent prognostic markers, such as MRI measures, may be helpful for patient stratification into clinical trials. It is also important that study end-points, such as survival are independent from demographic factors. [41].

\section{Limitations and future directions}

Our study outlines a prediction method based on singletime point MRI data, which is a snapshot of in vivo pathology at specific moment in the patient's disease trajectory.

Survival prediction may be more accurate if multiple time-points are included and longitudinal change over time is considered. [2] Moreover, the inclusion of other disease-specific anatomical regions, such as basal ganglia [44, 45], spinal cord [46, 47], cerebellar [48] or electrophysiological measures [49] may improve prognostic categorisation further. As only patients scanned at least 18 months ago were included, the sample size of the study is relatively limited and $20 \%$ of the patients were randomly allocated to the validation sample to demonstrate the generalizability of the methods. The present pilot study outlines a proposed prognostic algorithm which should ideally be replicated in larger cohorts or data pooled from multiple centres. Other future directions include assessment of two-year survival, or other clinical milestones, such as introduction of non-invasive ventilation, walking aids, feeding tubes etc. In this study, the cognitive and behavioural profile of the patients were not considered, despite evidence that executive dysfunction is associated with shorter survival [7] and compliance with assistive devices. [36] We also acknowledge that, with the current MR technology, the additional prognostic value of MRI indices is limited, and may not be substantial enough to be incorporated in clinical trial designs.

\section{Conclusions}

The combination of objective MRI measures and key clinical indices enable the accurate prediction of 18month survival in ALS. Accurate, objective and validated prognostic markers are urgently required in ALS, and have implications both for clinical trial designs and individualised patient care.

\section{Additional file}

\begin{abstract}
Additional file 1: Table S1. Coefficients estimates of each logistic ridge regression.Table S2. Clinical features. Demographic and clinical data of correctly and misclassified patients surviving less than 18 months of the training sample using a cut-off of 50\% probability. Table S3. Clinical features. Demographic and clinical data of correctly and misclassified patients surviving $>18$ months of the training sample using a cut-off of 50\% probability. Table S4. Clinical features. Demographic and clinical data of correctly and misclassified patients surviving $<18$ months of the validation sample using a cut-off of 50\% probability. Table S5. Clinical features. Demographic and clinical data of correctly and misclassified patients surviving $>18$ months of the validation sample using a cut-off of 50\% probability. Table S6. MRI features. Demographic and clinical data of correctly and misclassified patients surviving $<18$ months of the training sample using a cut-off of 50\% probability. Table S7. MRI features. Demographic and clinical data of correctly and misclassified patients surviving $>18$ months of the training sample using a cut-off of 50\% probability. Table S8. MRI features. Demographic and clinical data of correctly and misclassified patients surviving $<18$ months of the validation sample using a cut-off of 50\% probability. Table S9. MRI features. Demographic and clinical data of correctly and misclassified patients surviving $>18$ months of the validation sample using a cut-off of $50 \%$ probability. Table S10. Clinical and MRI features. Demographic and clinical data of correctly and misclassified patients surviving $<18$ months of the training sample using a cut-off of 50\% probability. Table S11. Clinical and MRI features. Demographic and clinical data of correctly and misclassified patients surviving $>18$ months of the training sample using a cut-off of $50 \%$ probability. Table S12. Clinical and MRI features. Demographic and clinical data of correctly and misclassified patients surviving $<18$ months of the validation sample using a cut-off of 50\% probability. Table S13. Clinical and MRI features. Demographic and clinical data of correctly and misclassified patients surviving $>18$ months of the validation sample using a cut-off of $50 \%$ probability. (DOCX $39 \mathrm{~kb}$ )
\end{abstract}

\section{Abbreviations}

AD: Axial diffusivity; ALS: Amyotrophic lateral sclerosis; ALSFRS-R: The revised ALS functional rating scale; CT: Cortical thickness; DTI: Diffusion tensor imaging; FA: Fractional anisotropy; FDR: False discovery rate; FWR: Family wise error; HC: Healthy control; MD: Mean diffusivity; MRI: Magnetic resonance imaging; RD: Radial diffusivity; WM: White matter 


\section{Acknowledgements}

We are grateful for the generosity and kindness of all of our patients and their caregivers who have kindly participated in this study.

\section{Funding}

The study was supported by the Irish Institute of Clinical Neuroscience IICN Novartis Ireland Research Grant (IICN-2016), the Iris O'Brien Foundation, the Perrigo Clinician-Scientist Research Fellowship, the Health Research Board (HRB), the Research Motor Neuron (RMN-Ireland) Foundation and the EU-Joint Programme for Neurodegeneration (JPND) SOPHIA project. The sponsors of the study had no role in study design, writing of the report or decision to submit this work for publication.

\section{Availability of data and materials}

Raw datasets are available on request from the corresponding author.

\section{Authors' contributions}

CS contributed to data collection, study design, analyses and drafting the manuscript. OH contributed to the clinical aspects of the study, study design and revision of the manuscript for intellectual content. PB contributed to data collection, study design, analyses and drafting the manuscript. All authors have read and approved the manuscript.

\section{Competing interests}

Ms. Christina Schuster reports no disclosures.

Prof. Hardiman has received speaking honoraria from Janssen Cilag, Biogen Idec, Sanofi Aventis and Merck-Serono, she has been a member of advisory panels for Biogen Idec, Allergen, Cytokinetics, Ono Pharmaceuticals and Sanofi Aventis.

Dr. Peter Bede reports no disclosures.

\section{Consent for publication}

Not applicable.

\section{Ethics approval and consent to participate}

All participants provided written informed consent in accordance to the Medical Ethics approval of the research project (Ethics (Medical Research) Committee - Beaumont Hospital, Dublin, Ireland).

\section{Publisher's Note}

Springer Nature remains neutral with regard to jurisdictional claims in published maps and institutional affiliations.

\section{Received: 22 September 2016 Accepted: 1 April 2017} Published online: 17 April 2017

\section{References}

1. Hardiman O, van den Berg LH, Kiernan MC. Clinical diagnosis and management of amyotrophic lateral sclerosis. Nat Rev Neurol. 2011;7(11): 639-49.

2. Schuster $C$, et al. Presymptomatic and longitudinal neuroimaging in neurodegeneration-from snapshots to motion picture: a systematic review. J Neurol Neurosurg Psychiatry. 2015:86(10):1089-96.

3. Byrne $\mathrm{S}$, et al. Cognitive and clinical characteristics of patients with amyotrophic lateral sclerosis carrying a C9orf72 repeat expansion: a population-based cohort study. Lancet Neurol. 2012;11(3):232-40.

4. Bede P, et al. Multiparametric MRI study of ALS stratified for the C9orf72 genotype. Neurology. 2013;81(4):361-9.

5. Mitsumoto $\mathrm{H}$, Brooks BR, Silani V. Clinical trials in amyotrophic latera sclerosis: why so many negative trials and how can trials be improved? Lancet Neurol. 2014;13(11):1127-38.

6. Gordon $\mathrm{PH}$, et al. Predicting survival of patients with amyotrophic lateral sclerosis at presentation: a 15-year experience. Neurodegener Dis. 2012; 12(2):81-90

7. Elamin $M$, et al. Executive dysfunction is a negative prognostic indicator in patients with ALS without dementia. Neurology. 2011;76(14):1263-9.

8. Wolf J, et al. Factors predicting one-year mortality in amyotrophic lateral sclerosis patients-data from a population-based registry. BMC Neurol. 2014;14:197

9. Chio A, et al. Prognostic factors in ALS: A critical review. Amyotroph Latera Scler. 2009;10(5-6):310-23.
10. Rooney J, et al. A multidisciplinary clinic approach improves survival in ALS: a comparative study of ALS in Ireland and Northern Ireland. J Neurol Neurosurg Psychiatry. 2015;86(5):496-501.

11. Turner MR, et al. Neuroimaging in amyotrophic lateral sclerosis. Biomark Med. 2012;6(3):319-37.

12. Bede $\mathrm{P}$, Hardiman O. Lessons of ALS imaging: Pitfalls and future directions A critical review. Neuroimage Clin. 2014;4:436-43.

13. Schuster $C$, et al. Longitudinal course of cortical thickness decline in amyotrophic lateral sclerosis. J Neurol. 2014;261(10):1871-80.

14. Bede $P$, et al. Grey matter correlates of clinical variables in amyotrophic lateral sclerosis (ALS): a neuroimaging study of ALS motor phenotype heterogeneity and cortical focality. J Neurol Neurosurg Psychiatry. 2013; 84(7):766-73.

15. Schuster $C$, et al. The segmental diffusivity profile of amyotrophic latera sclerosis associated white matter degeneration. Eur J Neurol. 2016;23(8): 1361-71.

16. Grolez $\mathrm{G}$, et al. The value of magnetic resonance imaging as a biomarker for amyotrophic lateral sclerosis: a systematic review. BMC Neurol. 2016;16(1):155

17. Orru G, et al. Using Support Vector Machine to identify imaging biomarkers of neurological and psychiatric disease: a critical review. Neurosci Biobehav Rev. 2012;36(4):1140-52.

18. Foerster BR, et al. Diagnostic accuracy of diffusion tensor imaging in amyotrophic lateral sclerosis: a systematic review and individual patient data meta-analysis. Acad Radiol. 2013;20(9):1099-106.

19. Ben Bashat $D$, et al. A potential tool for the diagnosis of ALS based on diffusion tensor imaging. Amyotroph Lateral Scler. 2011:12(6):398-405.

20. Welsh RC, Jelsone-Swain LM, Foerster BR. The utility of independent component analysis and machine learning in the identification of the amyotrophic lateral sclerosis diseased brain. Front Hum Neurosci. 2013;7:251.

21. Kalra S, et al. Cerebral degeneration predicts survival in amyotrophic lateral sclerosis. J Neurol Neurosurg Psychiatry. 2006;77(11):1253-5.

22. Agosta F, et al. MRI predictors of long-term evolution in amyotrophic lateral sclerosis. Eur J Neurosci. 2010;32(9):1490-6.

23. Brooks BR, et al. El Escorial revisited: revised criteria for the diagnosis of amyotrophic lateral sclerosis. Amyotroph Lateral Scler Other Motor Neuron Disord. 2000;1(5):293-9.

24. Lillo $\mathrm{P}$, et al. Grey and white matter changes across the amyotrophic lateral sclerosis-frontotemporal dementia continuum. PLoS One. 2012;7(8):e43993.

25. Bede $P$, et al. Sexual dimorphism in ALS: Exploring gender-specific neuroimaging signatures. In: Amyotroph Lateral Scler Frontotemporal Degener; 2013.

26. Smith SM, et al. Tract-based spatial statistics: voxelwise analysis of multisubject diffusion data. Neurolmage. 2006;31(4):1487-505.

27. Rosas HD, et al. Cerebral cortex and the clinical expression of Huntington's disease: complexity and heterogeneity. Brain. 2008;131(4):1057-68.

28. Salat $\mathrm{DH}$, et al. Thinning of the Cerebral Cortex in Aging. Cereb Cortex. 2004;14(7):721-30

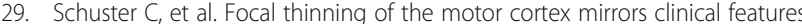
of amyotrophic lateral sclerosis and their phenotypes: a neuroimaging study. J Neurol. 2013;260(11):2856-64.

30. Verstraete $\mathrm{E}$, et al. Motor network degeneration in amyotrophic lateral sclerosis: a structural and functional connectivity study. PLoS One. 2010;5(10):-e13664.

31. Desikan RS, et al. An automated labeling system for subdividing the human cerebral cortex on MRI scans into gyral based regions of interest. Neurolmage. 2006:31(3):968-80.

32. Chiò A, Logroscino G. Prognostic factors in ALS: a critical review. Amyotrophic Lateral. 2009;10(5-6):310-23.

33. Koikkalainen J, et al. Improved classification of Alzheimer's disease data via removal of nuisance variability. PLoS One. 2012;7(2):e31112.

34. R Core Team, R: A language and environment for statistical computing. Vienna, Austria; 2014. URL http://www.R-project.org.

35. Friedman J, Hastie T, Tibshirani R. Regularization paths for generalized linear models via coordinate descent. J Stat Softw. 2010;33(1):1.

36. Olney RK, et al. The effects of executive and behavioral dysfunction on the course of ALS. Neurology. 2005;65(11):1774-7.

37. Connolly S, Galvin M, Hardiman O. End-of-life management in patients with amyotrophic lateral sclerosis. Lancet Neurol. 2015;14(4):435-42.

38. Bede $P$, et al. Palliative care in amyotrophic lateral sclerosis: a review of current international guidelines and initiatives. J Neurol Neurosurg Psychiatry. 2011;82(4):413-8. 
39. Nicholson KA, Cudkowicz ME, Berry JD. Clinical trial designs in amyotrophic lateral sclerosis: does one design fit all? Neurotherapeutics. 2015;12(2):376-83.

40. Bakkar N, Boehringer A, Bowser R. Use of biomarkers in ALS drug development and clinical trials. Brain Res. 2015;1607:94-107.

41. Beghi E, et al. The epidemiology and treatment of ALS: focus on the heterogeneity of the disease and critical appraisal of therapeutic trials. Amyotroph Lateral Scler. 2011;12(1):1-10.

42. Goutman SA, Feldman EL. Clinical Trials of Therapies for Amyotrophic Lateral Sclerosis: One Size Does Not Fit All. JAMA Neurol. 2015;72(7):743-4.

43. de Carvalho M, Swash M. Can selection of rapidly progressing patients shorten clinical trials in amyotrophic lateral sclerosis? Arch Neurol. 2006 63(4):557-60.

44. Bede $\mathrm{P}$, et al. Basal ganglia involvement in amyotrophic lateral sclerosis. Neurology. 2013;81(24):2107-15.

45. Machts J, et al. Basal ganglia pathology in ALS is associated with neuropsychological deficits. Neurology. 2015;85(15):1301-9.

46. Bede $\mathrm{P}$, et al. Spinal cord markers in ALS: diagnostic and biomarker considerations. Amyotroph Lateral Scler. 2012;13(5):407-15.

47. El Mendili MM, et al. Multi-parametric spinal cord MRI as potential progression marker in amyotrophic lateral sclerosis. PLoS One. 2014;9(4):e95516.

48. Bede $\mathrm{P}$, et al. Patterns of cerebral and cerebellar white matter degeneration in ALS. J Neurol Neurosurg Psychiatry. 2015;86(4):468-70.

49. de Carvalho $\mathrm{M}$, et al. Neurophysiological measures in amyotrophic lateral sclerosis: markers of progression in clinical trials. Amyotroph Lateral Scler Other Motor Neuron Disord. 2005;6(1):17-28.

\section{Submit your next manuscript to BioMed Central and we will help you at every step:}

- We accept pre-submission inquiries

- Our selector tool helps you to find the most relevant journal

- We provide round the clock customer support

- Convenient online submission

- Thorough peer review

- Inclusion in PubMed and all major indexing services

- Maximum visibility for your research

Submit your manuscript at www.biomedcentral.com/submit 